\title{
JOINED WING UAV MANEUVERABILITY
}

\author{
Dimo Zafirov \\ Associate.professor, TATT, Technical University of Sofia, Plovdiv branch, Bulgaria, zafirov@tu-plovdiv.bg
}

\begin{abstract}
Joined wing layout UAVs feature multiple advantages compared to the rest of UAVs. Their best advantage is the maneuverability. The report contains the analysis of the applicable UAV maneuvers and their application in various flight missions.
\end{abstract}

Keywords: UAV, Joined Wing, Maneuverability

$* * *$

\section{INTRODUCTION}

Studies related to ensuring of maneuver advantages to the modern jet fighters show the basic obstacles involve physical stamina of the pilots [1]. The advanced aerodymanics, robustness and control systems ensure designing of much higher maneuverability aircrafts provided they are unmanned. The sixth generation aircrafts are considered to be unmanned [2]. Advanced aerodynamic design, materials, ducts, avionics and software are to be utilized for their implementation.. An enormous research and development and experimental field of work is to be carried out initially until acceptable results are derived.

The use of Unmanned Aerial Vehicles (UAV) for surveilance and strike attacks involves also high maneuverability especially when flying civilian use missions.

The UAV civilian use missions for specific purposes (when extinguising fires, hail-storm cloud treatment and a strong trasverse ground missions) also involve high maneuverability.

The inclusion of the UAVs into the air traffic is an imminent task. Avoiding collisions with other objects shall be included as an essential requirement for the flying robots and not to be left to the pilots care. This also puts high demands on the maneuverability of UAVs.

Maneuverability is also an important issue for acrobatic aircraft. In this case the normal scheme rules; however, currently its potential seems to be exhausted.

All those abovementioned cases do not completely rule out the human factor involvement, however it will be used for solving non-standard situations. Man will be possible but not obligatory element in control loops.

The successful use of UAVs in recent years gives grounds to consider the psychological problems involved to have been eliminated.

\section{MANEUVERING OF AIRCRAFT - HISTORY, DEFINITIONS AND BASIC CONCEPTS}

Since the dawn of aviation maneuverability was the crucial quality, providing an advantage over the enemy.

Maneuver has been done mainly by means of aerodynamic handlebars to cause plane angular movement and subsequently modify the aerodynamic forces.

Maneuverability of the aircraft determines its ability to change the angular position and speed while controllability shows the ability to quickly provide the necessary attitude and thrust of the aircraft.

Fig. 1 shows the increase of the maneuvering and speed ranges of the aircraft. Field 1 is used by an aircraft without high maneuvering properties, field 2 is used by aircraft featuring high maneuverability properties, and field 3 is used by supermaneuverable UAVs only.

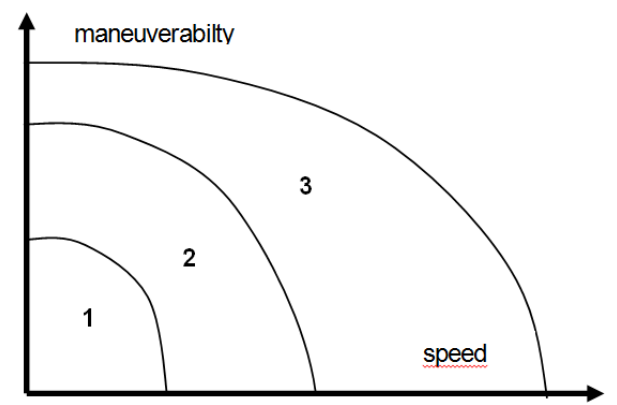

Fig - 1: Aircraft maneuverability fields

At present, the inability of humans to withstand high overloads is the main limit to increased maneuverability. New aircrafts involving supermaneuverabilty properties have emerged recently. This feature, according to the classical definition [3] 
is the ability of aircraft to be operated while exceeding the stalling angle. This is achieved due to the aerodynamic arrangement and mostly the controlled vector thrust. Supermaneuverability allows new stunt-flying, new offensive and defensive maneuvers and increasing the possibility of carrying out missions. The pilot is the most sensitive unit in the man-supermaneuverable aircraft system. Monitoring the target while attacking ground and air targets often takes the necessity of modifying the aerodynamic forces while not changing the angular position of the aircraft. This necessitated the creation of aircraft with the co-called direct control of aerodynamic forces.

Vertical take off and landing is a great advantage for either manned and unmanned aircraft. However, if they can quickly drop the speed to 0 and then quickly increase it during a flight that will result in unprecedented advantages either in attack and defense.

Guaranteed passage of the thrust vector through the center of mass regardless of its direction will give additional benefits associated with efficiency and maneuverability of an aircraft with such arrangement.

In several publications [1, 3-5] the term "mobility of the aircraft" (airframe agility) has been introduced, involving the properties of the aircraft to quickly modify the velocity vector and the spatial position.

Using all these solutions in an aircraft will result in pilots' inability to adequately control it and exploit its full capabilities.

For this reason the concept of aerodynamic arrangement of highly maneuverable aircraft being considered below is directed towards its unmanned implementation.

\section{HIGHLY MANEUVERABLE JOINED WING}

\section{AIRCRAFT WITH CONTROLLED VECTOR THRUST.}

An aircraft scheme featuring high maneuverability, reduced size and deformability, high strength, improved aerodynamics and performance and reduced run and run-on operations in [6].

The joined wing high maneuverability aircraft consists of a front and rear planes joined through a central load-carrying member while the wings ends are rigidly joined through lateral streamlined load-carrying members. The spatial load-carrying frame thus shaped from the central load-carrying member, wings and lateral streamlined load-carrying members affords the aircraft high rigidity and strain resistance.
The wings have lift devices incorporate leading edge flaps, rear edge flaps and ailerons that can be used in a combined way depending on the maneuver being performed. The rear edge flaps and ailerons can also function as elevator and turn rudder and for direct control of the lifting and lateral forces.

One or several engines are fixed jointly via a frame to the central load-carrying component, close to the aircraft mass centre.

The hinged engine is thus allowed to elongate to large angles while providing for $3 \mathrm{D}$ control of the thrust vector in the horizontal and vertical planes.

The simultaneous divergence of the lift devices of both wings allows for sharp increasing of the maximal aircraft bearing capacity that results in no supplementary aerodynamic moments to be balanced. The synchronised divergence of the lift devices can result in a significant expansion of the operational centerings scope.

The synchronised combined divergence of the rudders and engine allows for direct and indirect control of the aerodymanic forces and maneuvers being performed that involve sharp alteration of the motion direction and angular position of the aircraft.

The aircraft can take off and touch down as an usual one /with run-out and landing run/ and if sufficient thrust-to-weight ratio is available it can take off vertically. The aircratfts with vertical takeoff and tocuhdown usually involve more than one engine thus requiring precise thrust balancing at these modes which results in a more complicated structure. In most of the cases some of the engines are used in the takeoff and tochdown mode only thus making the structure more bulky. The aircraft in concern is capable of taking off with one engine only which also supports horizontal flight. The takeoff and touchdown stability can be secured with small divergences of the engine so that its thrust vector could always pass through the mass centre.

It is known that when the rudders are used as elevators and ailerons they are referred to as elevons [elevon=elev(ator)+(ailer)on]. When they are used as flaps and ailerons they are referred to as flaperons [flaperon=flap+(ail)eron].

With the joined wing scheme the rear edge flaps and ailerons can be used as elevators, slope rudders and direct control of the lifting and lateral forces thus sloping in various combinations. This could result in the introduction of a new term for combined wings such as flapeleron [flapeleron = flap+ele(vator)+(aile)ron]. 
The aircraft can be implemented in manned and unmanned versions. With the manned mode aircrafts with a much larger net load can be designed featuring equal dimensions compared to the existing ones hitherto.

In addition the joined wing along with the central loadcarrying member allow for very high overload maneuvers the unmanned aircrafts are involved with.

The streamlined lateral load-carrying members at the wings ends can be offset to the air flow thus providing for stabilty against divergences from course and slope. They can be furnished with rudders and direct control of the lateral force. The combined divergence of the rudders and the thrust vector by the control system affords the aircraft hypermaneuverability thus making it capable of performing maneuvers otherwise not possible for aircrafts with other schemes.

The net load can be placed in the centroplanes of the wings that form depending on the necessary volumes.

The joined wing high maneuverability aircraft advantages compared to equivalent aircrafts with other schemes are that it features better design, aerodynamic, dynamic, strength, mass and operational characteristics.

The technical solution being used reduces the necessary span and structure mass of the aircraft thus resulting in smaller span and tare weight compared to equivalent aircrafts with other schemes and ensuring advantages with air operation and maintenance.

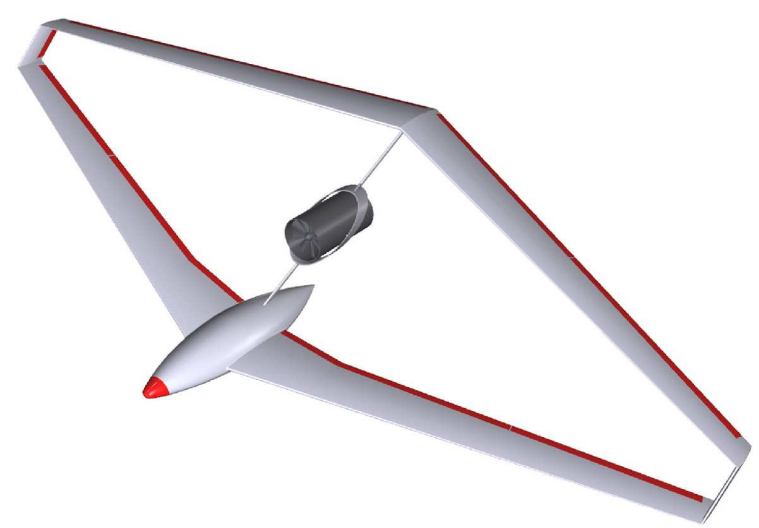

Fig 2: General view of a joined wing high maneuverability UAV

\section{A JOINED WING AND CONTROLLABLE}

\section{THRUST VECTOR AIRCRAFT MANEUVERS.}

It is evidently that the UAV scheme available involves much more maneuverability. It is due to the following:

- UAV can reduce their speed to zero, as lift in this case will be provided by the engine (i. e. no real failure modes);

- UAV can achieve much greater overloads while maneuvering (the specific scheme can be effortlessly designed to undergo loads up to and over $40 \mathrm{~g}$ );

- The diving maximal speed will be high for the lack of causes for flatter arising.

\section{RESULTS}

A great number of wind channel experiments on determination of the characteristics of the UAV have been carried out. The results are published in [5-7].

UAV JoWi 2 flight tests have started to observe good stability and controllability of the aircraft. The flight test program also includes the aircraft maneuverability characterisctics determination.

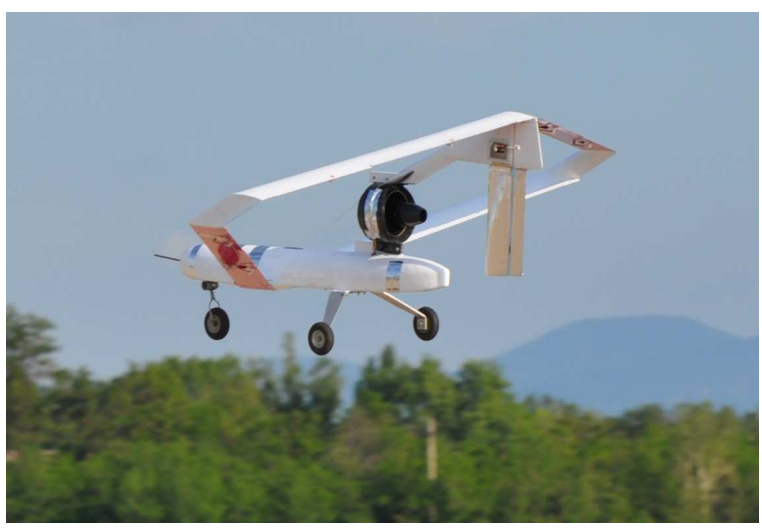

Fig 3: JoWi 2 testbed in flight

\section{CONCLUSIONS}

The joined wing and controllable thrust vector aircraft will feature high maneuverability and will outmatch the aircratfts with other schemes in both manned and unmanned versions.

An aircraft with the proposed scheme will allow you to maneuver without changing its angular position.

Maneuverability of such aircraft will allow you to carry out successful offensive and defensive maneuvers against enemy aircraft and missiles.

\section{REFERENCES}

[1] Patten V., Supermaneuverability and Superagility, Aeromedical and Training Digest, vol. 7, issue 1 (January 1993)

[2] Herbst, W., Supermanueverability, 1983, MesserschmittBolkow-Blohm GMBH: Ottoburn bei Munchen

[3] Practical limits of supermaneuverability and full envelope 
agility, AIAA 96-3493 Conference Proceedings (Aug. 1996)

[4] Blaye P. Agility: definitions, basic concepts, and history. ONERA, NATO/RTO TR-015. 2001.

[5] Zafirov D., A joined wing high maneuverability aircraft, Techsys '2009, Plovdiv

[6] Zafirov D., Panayotov H., Experimental results analysis of joined-wing aircrafts, BULTRANS Conference, Sozopol, Bulgaria, 2009

[7] Panayotov H., Zafirov D., Aerodynamic Optimization of Joined-Wing for Unmanned Aerial Vehicle, Journal of the Technical University at Plovdiv, "Fundamental Sciences and Applications", Vol. 13(8), Plovdiv, Bulgaria, 2006.

\section{BIOGRAPHIES}

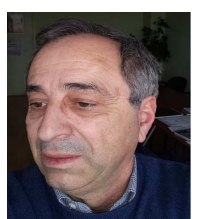

Assoc. prof. Dimo Zafirov $\mathrm{PhD}$ is lecturer in design of aircraft and has professional experience in the field of UAVs. His interests are focused on the study of aircraft with joined wing. 